\title{
Analisi dell'anisotropia microstrutturale in materiali compositi rinforzati con fibre corte
}

\author{
A. Bernasconi ${ }^{\text {a }}$, F. Cosmi ${ }^{\text {b }}$, D. Dreossi ${ }^{\text {c }}$ \\ ${ }^{a}$ Dipartimento di Meccanica, Politecnico di Milano, via La Masa 34 - 20156 Milano \\ ${ }^{b}$ Dipartimento di Ingegneria Meccanica, Università degli Studi di Trieste, via A. Valerio 10 - Trieste \\ ${ }^{c}$ Sincrotrone Trieste, Area Science Park, Basovizza, Trieste.
}

\begin{abstract}
RIASSUNTO. La microtomografia con luce di sincrotrone si è rivelata una tecnica particolarmente efficace per l'analisi della struttura risultante dalla distribuzione degli orientamenti assunti dalle fibre di rinforzo di compositi rinforzati con fibre di vetro. La ricostruzione dell'immagine tridimensionale ha consentito la visualizzazione della distribuzione spaziale delle fibre all'interno della matrice polimerica anche nel caso di fibre di piccole dimensioni (diametro medio di 10 micrometri). E' stato quindi possibile misurare le differenze nell'orientamento delle fibre nei differenti strati di un campione utilizzando metodi basati sulla valutazione del Mean Intercept Length (MIL) e del fabric tensor. La tecnica descritta è stata applicata a un campione di poliammide 6 rinforzato con il $30 \%$ di fibre corte di vetro ricavato da lastra.
\end{abstract}

ABSTRACT. Synchrotron light microtomography has proved to be particularly efficient in order to analyze the microstructural characteristics in terms of reinforce fibre distribution and orientation in glass fibre reinforced composites. The spatial distribution of fibre within the polymeric matrix could be detected even in case of fibre characterized by a small diameter (10 micrometers average diameter). Differences in orientation distribution within a sample could be measured using the Mean Intercept Length (MIL) and the fabric tensor. The results presented herein refer to a sample of a $30 \%$ by weight glass fibre reinforced polyamide 6 , extracted form a thin plate.

PAROLE CHIAVE. Compositi rinforzati con fibre corte; Anisotropia; Microtomografia; Mean intercept lenght.

\section{INTRODUZIONE}

I polimeri termoplastici rinforzati con fibre di vetro o di carbonio vengono sempre più frequentemente impiegati per la realizzazione di componenti soggetti a carichi anche rilevanti, grazie all'elevata resistenza specifica che ne consente l'impiego anche in sostituzione di materiali metallici.

L'effetto di rinforzo offerto dalle fibre consiste in un aumento di rigidezza e di resistenza, statica e a fatica, che dipende sia dal rapporto di forma (diametro/lunghezza della fibra) sia dall'orientamento delle fibre rispetto alla direzione lungo la quale agiscono gli sforzi [1-4].

Lo stampaggio a iniezione è il processo tecnologico più diffuso per la produzione di manufatti realizzati con tali materiali. Le fibre vengono dapprima aggiunte al polimero fuso durante un'estrusione. Tagliando gli estrusi si ottengono i granuli (pellet), che vengono utilizzati per alimentare la macchina per stampaggio a iniezione. In questa macchina, il polimero viene nuovamente fuso e le fibre, pertanto già inglobate nel materiale prima dello stampaggio, vengono trascinate dal flusso del polimero fuso nello stampo.

Il flusso di riempimento, il cui comportamento dinamico è assimilabile a quello di un fluido ad elevata viscosità, determina complesse interazioni che risultano:

- in una frantumazione, per cui la distribuzione delle lunghezze delle fibre assume l'andamento tipico di una distribuzione di Weibull, come indicato in Fig. 1(a) per un provino standard;

- in una forte influenza del comportamento dinamico del flusso sulla disposizione delle fibre, che si dispongono secondo orientamenti preferenziali, Fig. 1(b).

In particolare, il profilo di velocità del flusso presenta una zona a gradiente elevato in prossimità delle pareti dello stampo ed una zona a profilo tendenzialmente uniforme nella parte centrale dello spessore della cavità dello stampo. Di conseguenza, nei volumi di materiale che al centro sono soggetti principalmente a deformazioni trasversali, le fibre tendono a collocarsi in direzione perpendicolare al flusso di iniezione, mentre in prossimità delle pareti le fibre tendono a disporsi parallelamente al flusso, per effetto degli sforzi di taglio.

Le condizioni fluidodinamiche che si vengono a creare durante il processo di iniezione definiscono quindi gli orientamenti delle fibre (FOD, Fibre Orientaton Distribution) che a loro volta determinano le proprietà meccaniche. A titolo di esempio, in Fig. 2 si possono apprezzare le differenze di resistenza, rispettivamente statica (a) ed a fatica (b), che i provini standard, caratterizzati dall'assenza dello strato centrale con fibre perpendicolari all'asse, presentano rispetto a provini aventi la stessa frazione volumetrica in fibre, ma ricavati da lastra iniettata di testa, che presentano invece un marcato strato centrale. 


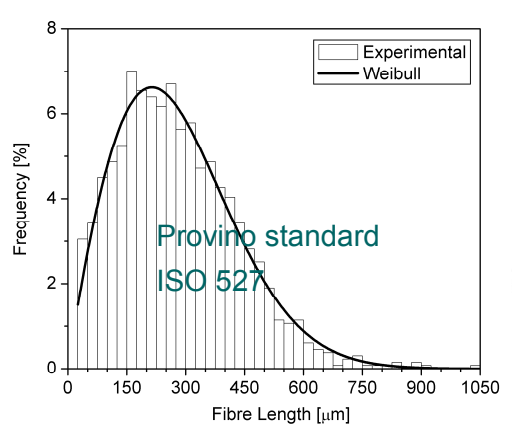

(a)

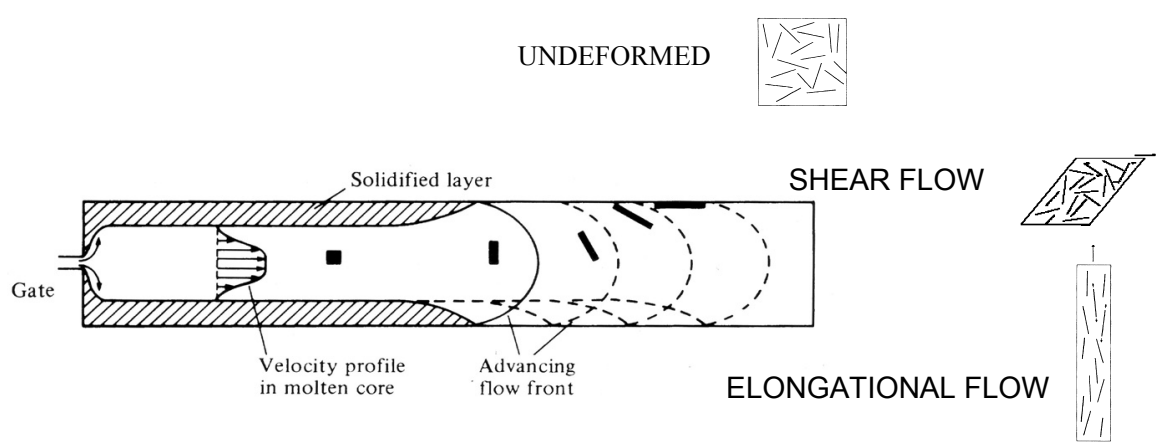

(b)

Figura 1: Distribuzione delle lunghezze [2] (a) e degli orientamenti (b) delle fibre nel processo di iniezione [5].
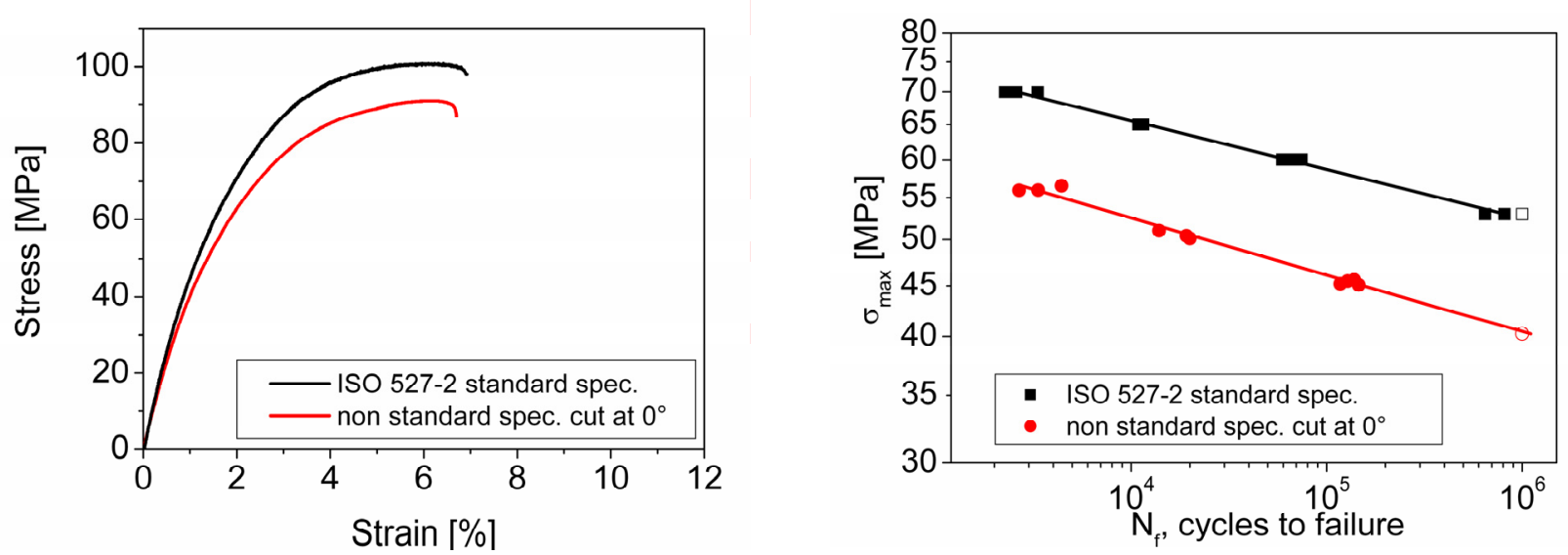

Figura 2: Resistenza statica (a) e a fatica (b) in provini standard e ricavati da lastra iniettata di testa [2].

La caratterizzazione delle distribuzioni di lunghezze e orientamento delle fibre costituisce quindi il primo passo per poter trasferire i risultati delle prove eseguite su provini standard a componenti reali, che inevitabilmente presentano geometrie più complesse.

Se da una parte la determinazione della distribuzione delle lunghezze delle fibre appare relativamente semplice, in quanto è sufficiente separarle dalla matrice polimerica per analizzarle successivamente al microscopio, più complessa appare la caratterizzazione della distribuzione degli orientamenti.

Da un punto di vista teorico, Advane e Tucker [6] hanno introdotto una formulazione tensoriale per la previsione delle orientazioni assunte dalle fibre, ipotizzate di lunghezza e diametro uniforme, in seguito al moto viscoso all'interno della matrice polimerica.

La tecnica sperimentale più diffusa è distruttiva e consiste nell'analisi di una serie di sezioni di un campione di materiale opportunamente preparato al microtomo $[7,8]$. L'orientazione viene ricostruita a partire dall'eccentricità della traccia ellittica della sezione della fibra. Questa tecnica presenta un'elevata incertezza nella misura degli angoli di orientamento, legata da un lato alle difficoltà nella preparazione del campione a causa della inevitabile frantumazione dei bordi delle fibre, dall'altro alla necessità di analizzare più sezioni adiacenti per discriminare tra i diversi angoli che possono corrispondere ad un'unica trac- cia. Lo sviluppo di tecniche di microscopia confocale [9] ha permesso in parte di superarne in parte le limitazioni, permettendo di focalizzare piani sottostanti il piano di sezione, fino ad una profondità di $150 \mu \mathrm{m}$, mantenendo tuttavia la necessità di sezionare il campione nella zona d'interesse.

Le tecniche radiografiche non sono distruttive, ma permettono di analizzare solo la proiezione delle fibre sul piano della lastra [10], per cui l'analisi risulta corretta solo per distribuzioni piane di fibre. Per ottenere una ricostruzione tridimensionale completa è necessario ricorrere a tecniche di microtomografia computerizzata [11]. Anche in questo caso, però, un approccio legato all'analisi diretta di ogni singola fibra è possibile solo se le fibre sono relativamente poco numerose e di diametro sufficientemente grande rispetto alla risoluzione del sensore.

Come descritto in questa memoria, nella particolare applicazione al caso di compositi rinforzati con fibre di vetro, la microtomografia con luce di sincrotrone si è rivelata una tecnica particolarmente efficace, consentendo l'impiego di tecniche radiografiche in contrasto di fase, in grado di rendere visibili anche piccole disomogeneità all'interno del campione. La ricostruzione dell'immagine tridimensionale ha consentito la visualizzazione della distribuzione spaziale delle fibre all'interno della matrice polimerica anche nel caso di fibre di piccole dimensioni (diametro medio di 10 micrometri). E' stato quindi possi- 


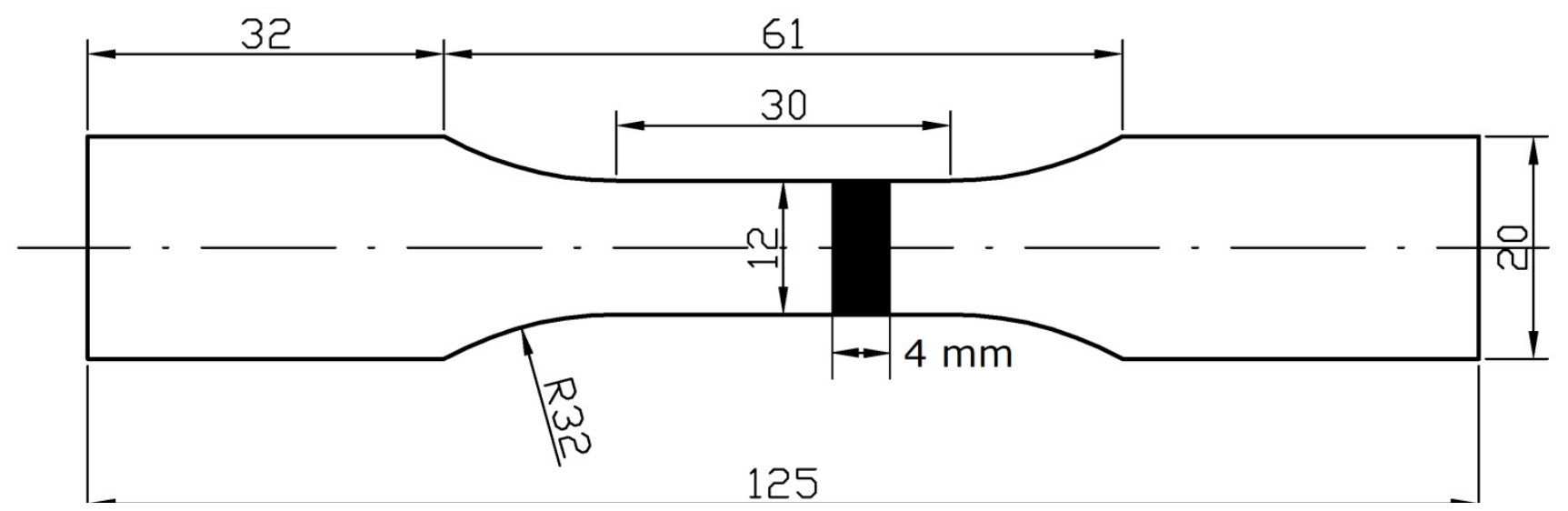

Figura 3: Provino, di spessore $3.2 \mathrm{~mm}$, da cui è stato ricavato il campione di materiale analizzato

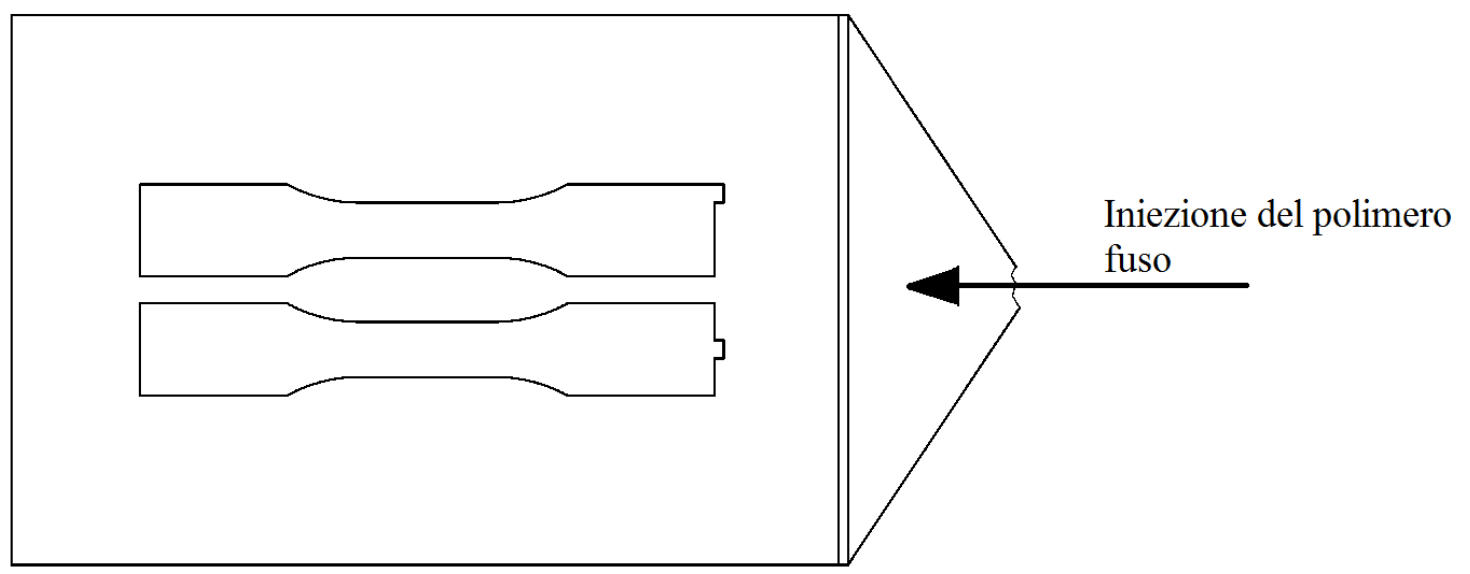

Figura 4: Lastra da cui è stato estratto il provino

bile misurare le differenze nell'orientamento dei differenti strati di un campione utilizzando metodi comunemente impiegati in altri settori, quali la geologia [12] e biologia [13], basati sulla valutazione di parametri legati alla distribuzione di fasi diverse in un volume, come ad esempio il Mean Intercept Length (MIL) o il fabric tensor, che sono stati applicati a un campione di poliammide 6 rinforzato con il $30 \%$ di fibre corte di vetro.

\section{MATERIALI E METODI}

\subsection{Campione}

Il materiale analizzato è un composito a matrice polimerica termoplastica, poliammide 6 , in cui il rinforzo è ottenuto mediante miscela del $30 \%$ in peso di fibre di vetro di tipo E aventi diametro medio di $10 \mu \mathrm{m}$ (PA6 GF 30). Come già citato, la lunghezza delle fibre presenta una distribuzione di tipo Weibull. Il valore medio della lunghezza delle fibre dipende dalle condizioni di stampaggio, dalla complessità dello stampo e dalla concentrazione di fibre e oscilla generalmente tra i 200 e i $300 \mu \mathrm{m}$, mentre le lunghezze massime possono raggiungere anche i $1,5 \mathrm{~mm}$.

Il campione oggetto delle misure è un parallelepipedo di base $3,2 \mathrm{~mm} \times 4 \mathrm{~mm}$ e altezza $12 \mathrm{~mm}$ estratto, come ri- portato in Fig.3, da un provino a sua volta ricavato una lastra iniettata di testa come indicato in Fig.4, di dimensioni 120 x $180 \mathrm{~mm}$ e $3,2 \mathrm{~mm}$ di spessore, utilizzata per le prove di fatica descritte in [2].

In queste condizioni è noto che le fibre nella zona centrale del provino (core) tendono ad orientarsi perpendicolarmente al flusso d'iniezione, mentre ai lati di questa (shell), lungo quasi tutto lo spessore, la disposizione delle fibre è parallela al flusso d'iniezione, come rappresentato schematicamente in Fig.5a sulla base dell'immagine ottenuta al microscopio ottico di una sezione perpendicolare al flusso d'iniezione. Infatti, come è possibile osservare in Fig.5b, in quasi tutta la sezione del provino la traccia delle fibre nel piano di sezione è approssimativamente ellittica o circolare, ad indicare che le fibre sono state intersecate perpendicolarmente dal piano di taglio, mentre in uno strato centrale di circa $200 \mu \mathrm{m}$ di altezza sono ben visibili numerose fibre parallele al piano di sezione.

Nelle lastre, caratterizzate da una larghezza della sezione trasversale molto maggiore rispetto allo spessore, le condizioni fluidodinamiche durante l'iniezione sono molto diverse da quelle che si verificano durante l'iniezione del provino con geometria standard, in cui le superfici dello stampo sono molto più vicine tra loro. La diversa struttura assunta dalle fibre di rinforzo in conseguenza delle diverse condizioni del flusso di iniezione determina diffe- 


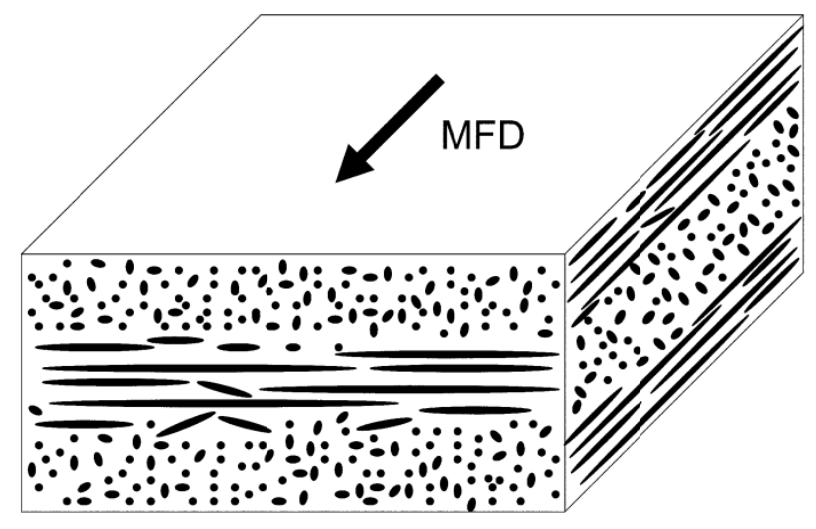

(a)

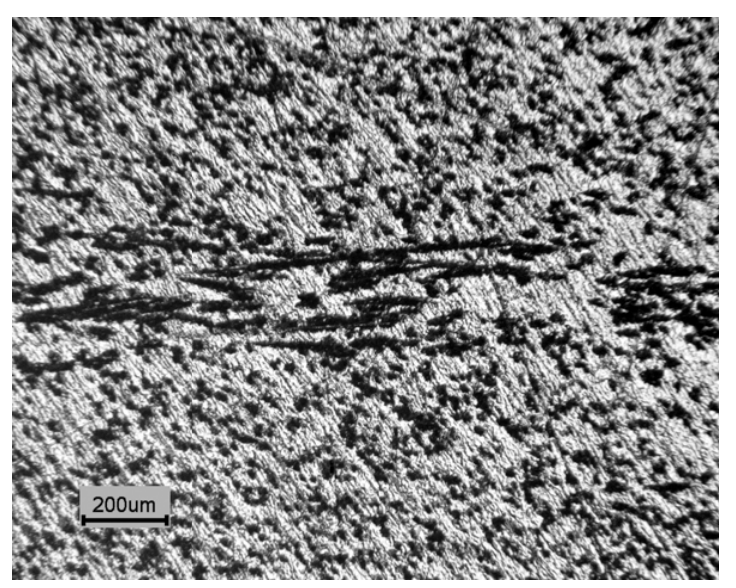

(b)

Figura 5: Campione di PA6 GF 30 estratto da una lastra di 3,2 $\mathrm{mm}$ di spessore: (a) schema della distribuzione delle fibre (MDF = direzione del flusso d'iniezione) e (b) particolare della distribuzione delle fibre nella zona centrale da immagine ottenuta al microscopio ottico di una sezione perpendicolare al flusso d'iniezione (fibre in nero).

\begin{tabular}{cccc}
\hline Campione & $\begin{array}{c}\mathrm{R}_{\mathrm{m}} \\
{[\mathrm{MPa}]}\end{array}$ & $\begin{array}{c}\mathrm{E} \\
{[\mathrm{MPa}]}\end{array}$ & $\begin{array}{c}\text { Deformazione a rottura } \\
{[\%]}\end{array}$ \\
\hline ISO 527-2 & $104.8( \pm 0.6)$ & $5846( \pm 139)$ & $6.78( \pm 0.15)$ \\
\hline Estratto da lastra & $89.5( \pm 3.6)$ & $4607( \pm 114)$ & $6.47( \pm 0.73)$ \\
\hline
\end{tabular}

Tabella 1 - Valori di resistenza a trazione, modulo elastico e allungamento a rottura di campioni di PA6GF30: confronto tra provino standard ISO 527-2 e provino estratto da lastra iniettata di testa.

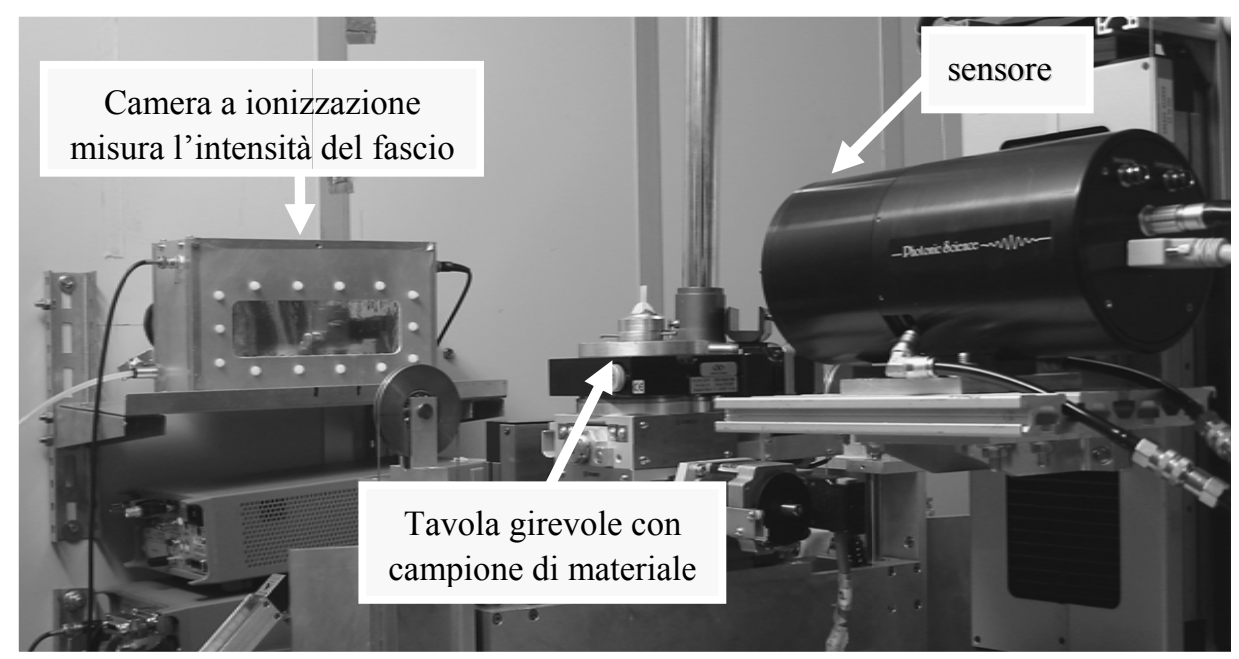

Figura 6: Set-up sperimentale per microtomografia con camera a ionizzazione all'uscita del fascio di raggi X, tavola girevole con un campione di materiale, rivelatore.

renze nelle proprietà meccaniche, come riportato in Tab. 1. La caratterizzazione dell'orientamento delle fibre rappresenta pertanto il primo passo per poter predire e modellare rigidezza e resistenza del composito nel componente reale.

\subsection{Acquisizione dati}

La micrografia con radiazione di sincrotrone è una tecnica ideale per l'analisi sperimentale della microstruttura di materiali compositi rinforzati con fibre corte, essendo libera dalle limitazioni tipiche degli approcci tradizionali menzionati nell'introduzione.
Le analisi sono state condotte presso la linea di luce SYRMEP, installata presso il sincrotrone di Trieste (Elettra) e dedicata all'imaging con raggi $\mathrm{X}$ monocromatici con energia selezionabile fra i 9 ed i $35 \mathrm{keV}$.

Il fascio originato da uno dei magneti curvanti di Elettra viene reso monocromatico da una doppia riflessione su cristalli di $\mathrm{Si}(111)$ e raggiunge la stazione sperimentale (Fig. 6), che ospita campione e rivelatore.

L'alta coerenza spaziale della sorgente consente di applicare tecniche innovative che utilizzano anche l'informazione legata alle variazioni di fase subite dalla radiazione nell'attraversare il campione, che consentono di mettere 


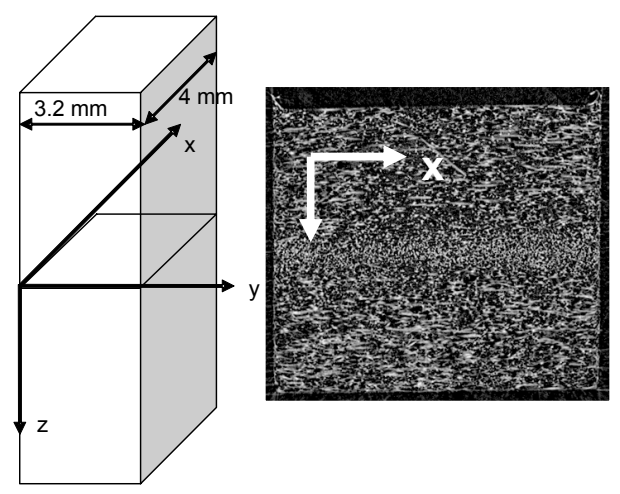

Ricostruzione tomografica 2D
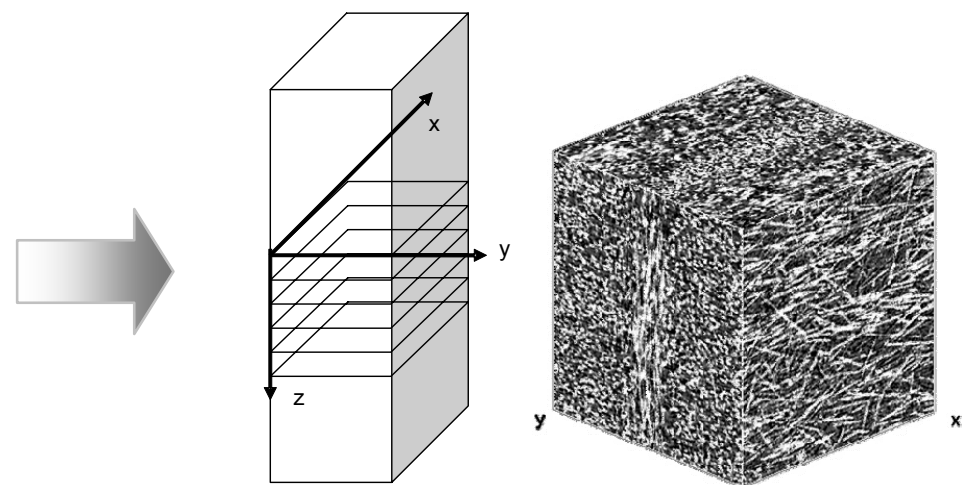

Volume 3D

Figura 7: Ricostruzione tridimensionale di un volume cubico di lato 1,8 $\mathrm{mm}$, a partire dalle ricostruzioni tomografiche $2 \mathrm{D}$. Le fibre appaiono di colore chiaro.

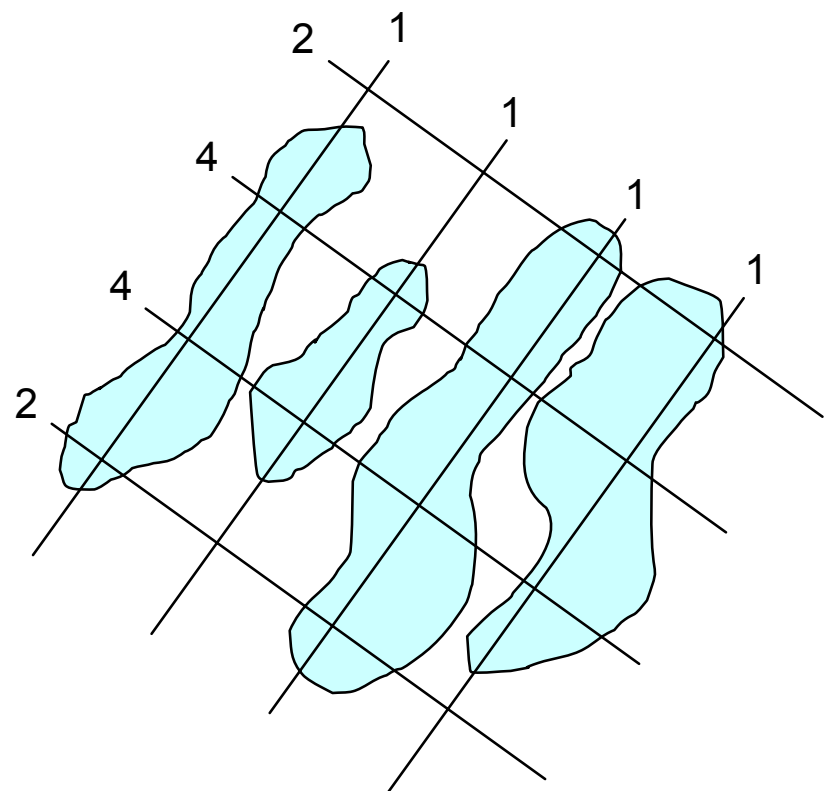

Figura 8: Conteggio delle intersezioni tra linee della griglia e passaggi di fase fibra-matrice per due diversi orientamenti della griglia di misura.

particolarmente in evidenza i bordi di disomogeneità anche molto piccole.

La radiografia convenzionale registra le proprietà di assorbimento dei raggi $\mathrm{X}$ da parte di un oggetto. Il contrasto dell'immagine è generato dalle differenze di densità, composizione e spessore del campione e si basa esclusivamente sul rilievo della variazione di ampiezza dei raggi $\mathrm{X}$ che lo attraversano. Il limite principale di questa tecnica consiste nel basso contrasto intrinseco dei materiali a basso numero atomico (per esempio i tessuti molli) o, più in generale, dei materiali con piccole differenze di assorbimento da punto a punto.

In generale, quando la sorgente di raggi X è caratterizzata da un elevata coerenza spaziale, come è il caso delle sorgenti di luce di sincrotrone, è possibile utilizzare anche l'informazione legata alle variazioni di fase che la radiazione subisce attraversando un oggetto. Questi effetti, soprattutto a basse energie, sono preponderanti rispetto a quelli connessi all'assorbimento e rendono visibili anche piccole disomogeneità all'interno del campione.

Numerosi approcci alla radiologia phase-sensitive sono stati riportati in letteratura e, tra questi, la radiografia in contrasto di fase (PHC, PHase Contrast) si caratterizza per la sua semplicità di implementazione: è sufficiente, infatti, porre il rivelatore ad una opportuna distanza dal soggetto da analizzare $[14,15]$. La linea di luce è equipaggiata per la microtomografia computerizzata ad alta risoluzione (micro-CT), una delle più avanzate tecniche non distruttive di indagine, in grado di visualizzare la struttura interna di diversi materiali [16]. Questa tecnica consiste nell'acquisire un grande numero di proiezioni radiografiche PhC (nel nostro caso 720) ottenute a diverse posizioni angolari del campione, collocato su una tavola rotante rispetto alla sorgente, fino a coprire una rotazione totale di $180^{\circ}$. Applicando un algoritmo basato sul metodo della retro-proiezione filtrata, dalle proiezioni angolari ottenute si ricostruiscono le diverse sezioni trasversali 

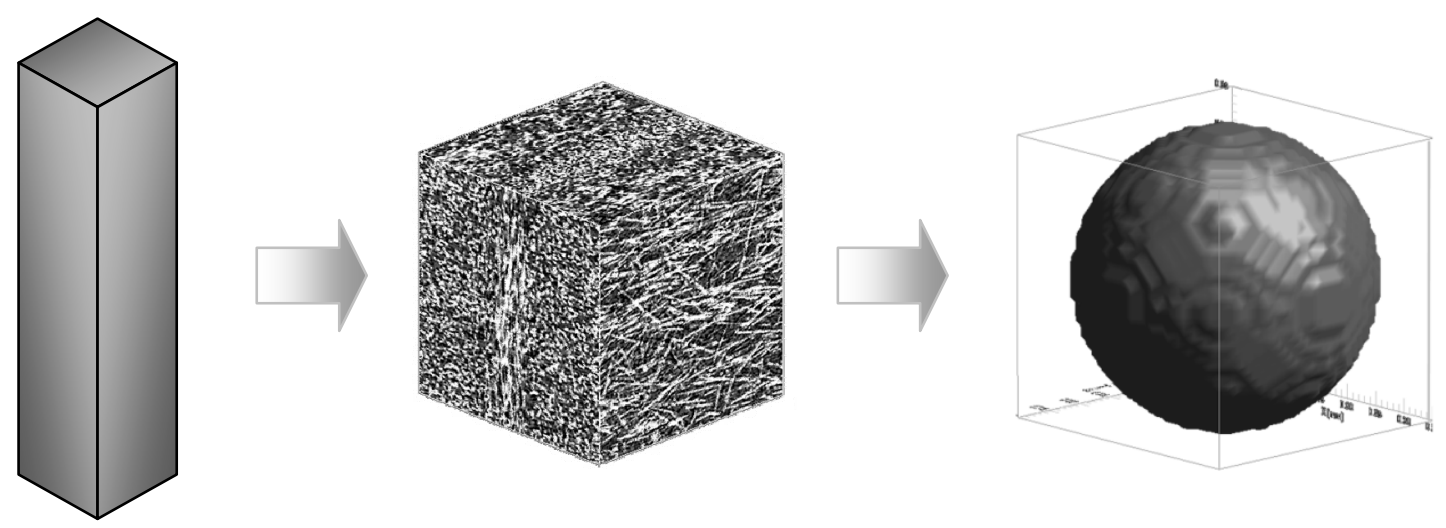

Figura 9: Volume sferico per l'analisi del MIL, estratto dal volume del campione ricostruito.

(slices) che riproducono la struttura del campione. Combinando una serie di slices si ottiene una rappresentazione volumetrica (Fig.7).

Rispetto ad una sorgente convenzionale, la luce di sincrotrone consente di migliorare la qualità dell'immagine, evitando effetti legati all'indurimento del fascio e consentendo misure quantitative sul campione in esame. Il rivelatore disponibile presso la stazione sperimentale della linea di luce SYRMEP è una CCD camera (Photonic Science XDI-VHR 1:2), 4008*2672 pixels di lato $9 \mu \mathrm{m}$ accoppiata mediante un taper di fibre ottiche ad uno schermo di ossisolfuro di gadolinio dello spessore di circa 5 micron 12 bit. La massima risoluzione spaziale attualmente ottenibile, pari circa alla dimensione del pixel, si posiziona al limite inferiore delle possibilità di individuazione delle fibre di rinforzo (vetro o carbonio). Queste risultano però ben evidenziate nelle tomografie grazie all'impiego, della radiografia in contrasto di fase.

\subsection{Analisi dell'anisotropia}

La quantificazione delle differenze nella disposizione delle fibre evidenziate dalla ricostruzione del campione passa attraverso l'analisi delle caratteristiche di anisotropia del volume analizzato.

$\mathrm{Nel}$ campione esaminato, dimensioni e numerosità fibre rendono difficilmente percorribile un approccio basato sull'analisi diretta di ogni singola fibra, come quello seguito da Shen et al. [11] per fibre relativamente poco numerose e con diametri dell'ordine dei $100 \mu \mathrm{m}$. Si è quindi preferito caratterizzare globalmente l'anisotropia del materiale, rinunciando a determinare l'orientamento di ogni singola fibra.

In letteratura non esiste una definizione univoca di misura dell'anisotropia, ma vengono suggeriti diversi metodi che fanno riferimento a grandezze diverse: a seconda del parametro che viene utilizzato, il risultato ottenuto può risultare più o meno adatto a caratterizzare la struttura esaminata [17].

La caratterizzazione proposta in questo lavoro utilizza un parametro, il Mean Intercept Length (MIL), comunemente impiegato in biomeccanica [13], che per le sue caratteristiche appare particolarmente promettente per descrivere la disposizione spaziale di fibre [18].
Il Mean Intercept Length, MIL, è la distanza media tra le interfacce delle due fasi di una struttura. Il principio alla base di questa misura consiste nel posizionare una griglia piana di lunghezza $L$ ed orientamento $\theta$ sulla struttura bifase e contare il numero $I$ di intersezioni tra le linee della griglia ed i passaggi di fase, nel nostro caso fibra-matrice, come illustrato in Figura 8.

Il $M I L$ è quindi dato da

$$
\operatorname{MIL}(\theta)=\frac{L}{I(\theta)}
$$

ed è funzione dell'orientamento della griglia lungo la quale viene effettuata la misura. Il valore di MIL sarà dunque più alto in corrispondenza della direzione preferenziale di orientamento delle fibre, che corrisponde ad un minor numero di passaggi di fase.

Ripetendo la misura $\mathrm{n}$ volte al variare dell'orientazione della griglia nello spazio, si possono rappresentare gli $n$ MIL associati alle diverse direzioni in un diagramma polare. Per i materiali a due componenti è possibile approssimare il luogo descritto con un ellissoide e quindi definire una rappresentazione equivalente a un tensore definito positivo del secondo ordine, chiamato il fabric tensor del $M I L$. Gli autovettori di questo tensore rappresentano le direzioni principali dell'anisotropia, mentre gli autovalori, $T_{1}, T_{2}, T_{3}$, costituiscono una misura della distribuzione della struttura intorno a queste direzioni. Per poter confrontare tra loro i risultati ottenuti in diversi campioni, $\mathrm{i}$ valori degli auto valori sono normalizzati imponendo che l'invariante primo sia unitario, vale a dire $T_{1}+T_{2}+T_{3}=$ 1. Un ulteriore parametro, utile per valutare quanto la struttura sia in effetti orientata in una direzione preferenziale, è il Degree of Anisotropy $D A=T_{3} / T_{1}$.

L'analisi dell'anisotropia mediante $M I L$ è influenzata da diversi fattori che vengono discussi nel seguito. Il software utilizzato è Quant3D [19].

\section{Volumi di interesse}

L'analisi del $M I L$ è stata eseguita all'interno di diversi volumi sferici estratti dal volume ricostruito come illustrato in Fig.9.

I volumi analizzati sono porzioni del volume originale ricostruito, estratti rispettivamente nelle zone shell e core. 

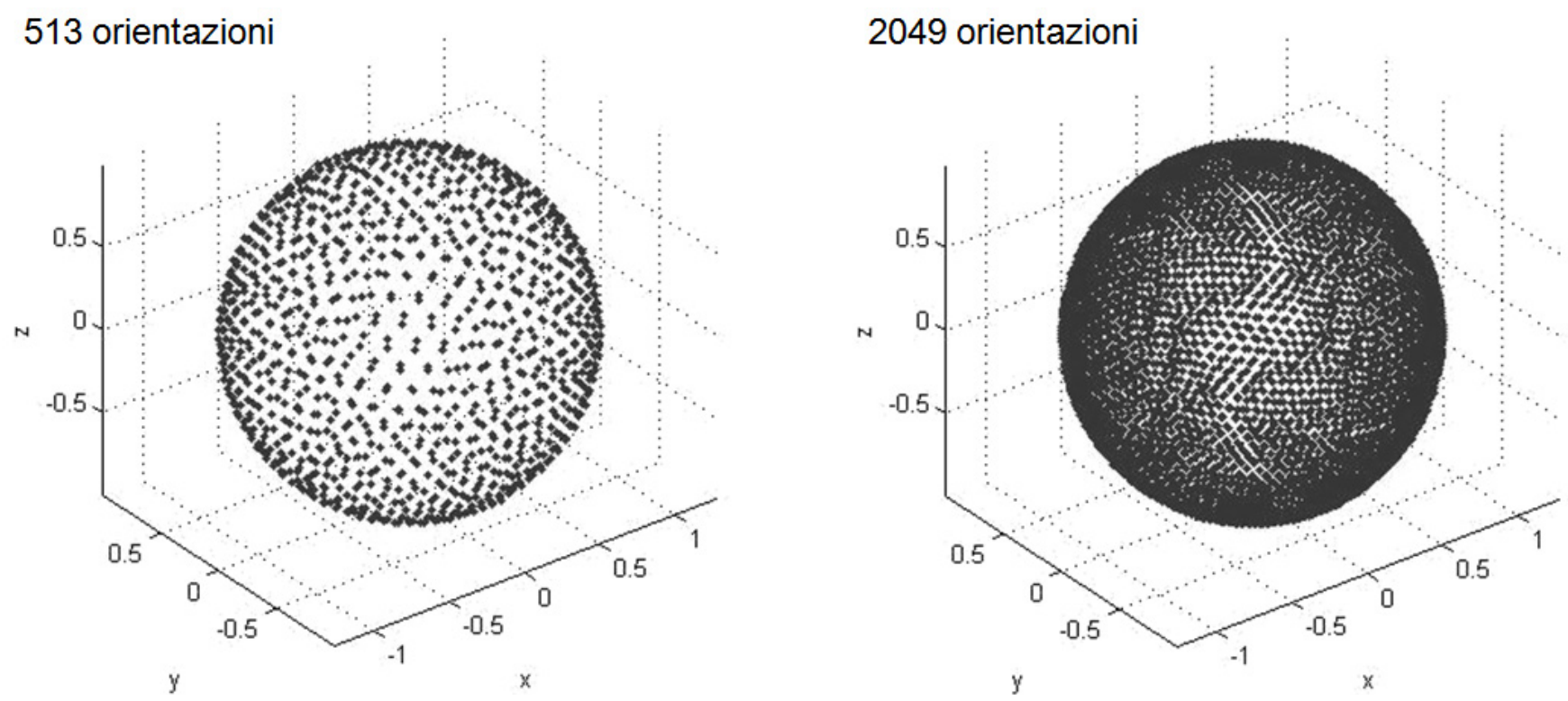

Figura 10: Proiezione delle direzioni di misura sul volume sferico per l'analisi del MIL.

\begin{tabular}{|c|c|c|c|c|c|c|}
\hline \multirow[b]{2}{*}{ prova 1} & \multirow{2}{*}{$\begin{array}{l}\text { DA } \\
1.82 \\
\end{array}$} & \multirow[b]{2}{*}{$T_{1}$} & \multicolumn{2}{|r|}{$\cos \alpha$} & \multirow{2}{*}{$\begin{array}{c}\cos \beta \\
-0.000382 \\
\end{array}$} & \multirow{2}{*}{$\begin{array}{c}\cos \gamma \\
0.054215 \\
\end{array}$} \\
\hline & & & 0.436245 & 0.998529 & & \\
\hline & & $T_{2}$ & 0.324459 & 0.00035 & 0.999038 & 0.000612 \\
\hline & & $T_{3}$ & 0.239296 & -0.05422 & -0.00059 & 0.998529 \\
\hline \multirow[t]{3}{*}{ prova 2} & 1.82 & $T_{1}$ & 0.436202 & 0.997874 & -0.04446 & 0.047642 \\
\hline & & $T_{2}$ & 0.323492 & 0.046186 & 0.998295 & -0.03569 \\
\hline & & $T_{3}$ & 0.240306 & -0.04597 & 0.037811 & 0.998227 \\
\hline \multirow[t]{3}{*}{ prova 3} & 1.80 & $T_{1}$ & 0.436052 & 0.998102 & -0.01715 & 0.059143 \\
\hline & & $T_{2}$ & 0.32189 & 0.018157 & 0.999698 & -0.01656 \\
\hline & & $T_{3}$ & 0.242058 & -0.05884 & 0.017604 & 0.998112 \\
\hline
\end{tabular}

Tabella 2 - Influenza della rotazione random iniziale (griglia di misura con 513 orientazioni e 1000 linee).

La dimensione del volume di interesse (VOI), ove non diversamente specificato, è di $40 \times 40 \times 40$ voxel $^{3}$, pari a $360 \times 360 \times 360 \mu \mathrm{m}^{3}$.

\section{Segmentazione}

Per poter eseguire le misure di $M I L$, è necessario identificare le due fasi, fibra e matrice, all'interno del volume di interesse, processo che viene indicato con il termine di segmentazione dell'immagine digitale.

In generale non esiste un unico algoritmo di segmentazione, ma è necessario scegliere la tecnica migliore per il problema specifico.

Nel caso del campione analizzato in questo lavoro, è noto dal processo di fabbricazione che la frazione volumetrica delle fibre all'interno della matrice, FV/TV (Fibre Volume/Total Volume), è del $15.7 \%$. E' stato per semplicità adottato un semplice algoritmo a soglia, posizionando il valore della soglia in modo tale da restituire il valore atteso di FV/TV nell'analisi dell'intero volume del campione ricostruito $\left(396 \times 343 \times 431\right.$ voxel $^{3}$, pari a $\left.3.564 \times 3.087 \times 3.879 \mathrm{~mm}^{3}\right)$.
Per le successive analisi delle diverse porzioni del medesimo campione che costituiscono i volumi di interesse, è stato sempre adottato lo stesso valore di soglia, determinato nel modo precedentemente descritto. In questo modo è stato anche possibile rilevare addensamenti e rarefazioni delle fibre nelle diverse zone del campione, che appaiono come variazioni locali del valore di FV/TV.

\section{Campionamento delle orientazioni}

Il risultato dell'analisi del MIL dipende dal numero di orientazioni della griglia utilizzata per la misura. Il software adottato consente all'utente di scegliere tra due possibilità, rispettivamente 513 e 2049 rotazioni, distribuite uniformemente sulla sfera impiegata per la misura, come illustrato in Fig. 10.

Per minimizzare gli errori sistematici, il software impone al set di orientazioni predeterminato una rotazione casuale iniziale. L'influenza della rotazione random iniziale sul risultato della misura è riportata in Tab.2, che riporta $\mathrm{i}$ parametri del fabric tensor del MIL ottenuti in tre successive misure eseguite nella zona shell del campione, adot- 

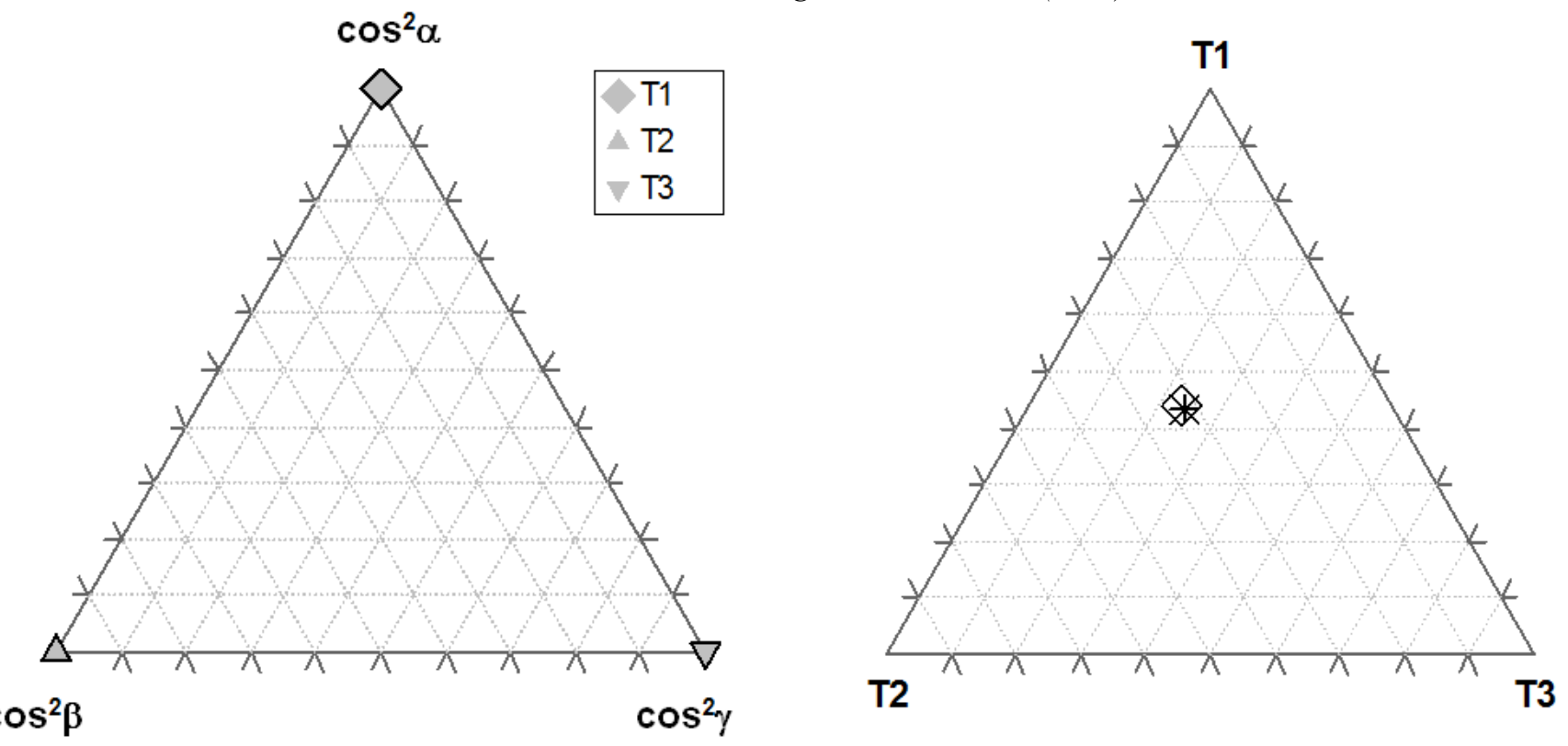

Figura 11: Rappresentazione dei parametri del fabric tensor del MIL in grafici ternari (griglia di misura con 513 orientazioni e 1000 linee).

\begin{tabular}{l|c|c|c|} 
& \multicolumn{3}{|c|}{$\begin{array}{c}\text { Degree of Anisotropy } D A \\
\text { (tempo di calcolo) }\end{array}$} \\
& 500 linee & 1000 linee & 2000 linee \\
\hline 513 orientazioni & $\mathbf{1 . 8 2}$ & $\mathbf{1 . 8 1}$ & $\mathbf{1 . 8 1}$ \\
& $(\mathrm{t}=13 \mathrm{~s})$ & $(\mathrm{t}=24 \mathrm{~s})$ & $(\mathrm{t}=48 \mathrm{~s})$ \\
\hline 2049 orientazioni & $\mathbf{1 . 8 1}$ & $\mathbf{1 . 8 1}$ & $\mathbf{1 . 8 1}$ \\
$(\mathrm{t}=48 \mathrm{~s})$ & $(\mathrm{t}=94 \mathrm{~s})$ & $(\mathrm{t}=188 \mathrm{~s})$ \\
\hline
\end{tabular}

Tabella 3 - Influenza del numero di orientazioni e di linee misura: $D A$ e tempi di calcolo.
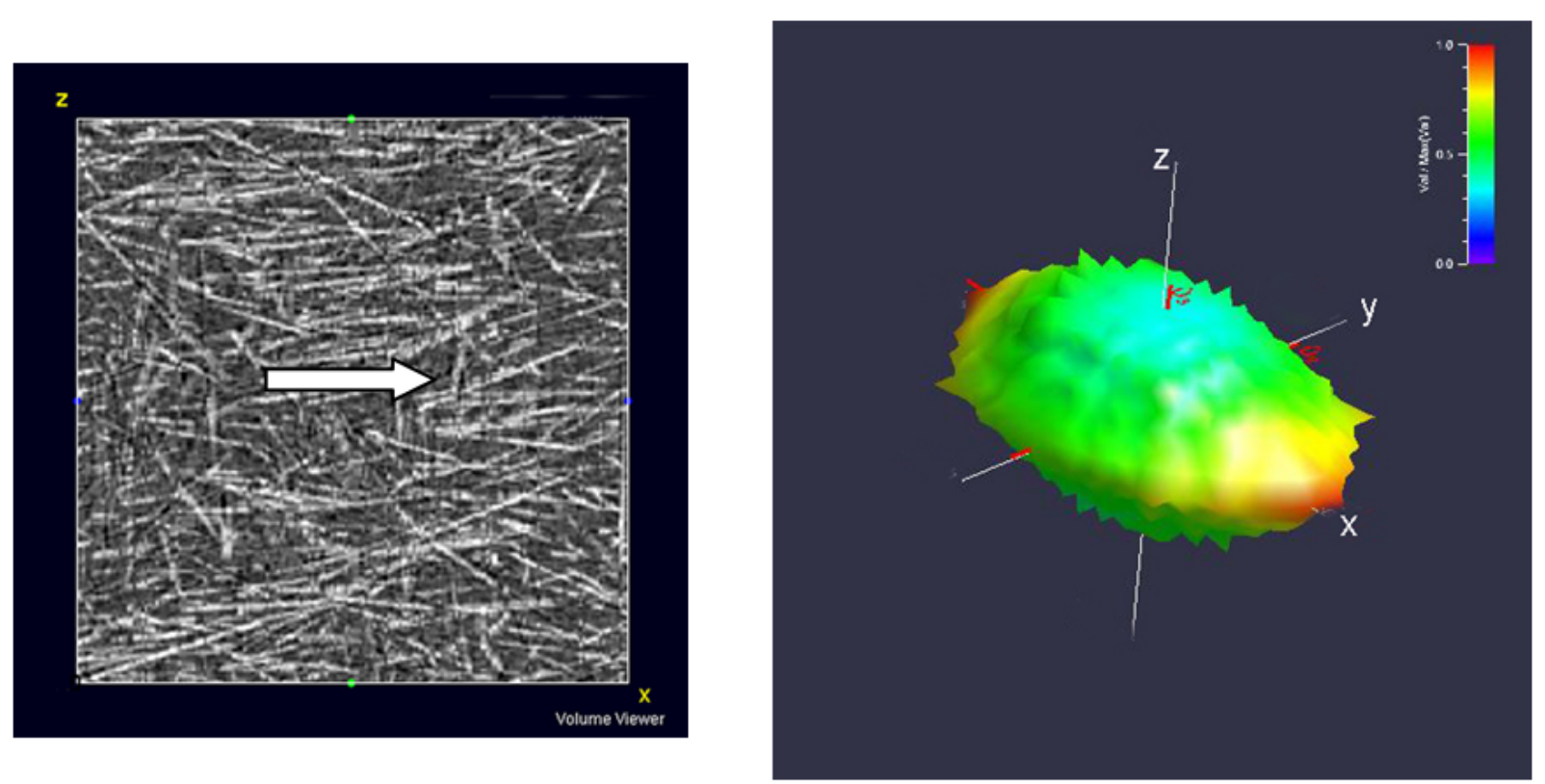

Figura 12: Zona shell - Ricostruzione tomografica della distribuzione delle fibre (la freccia indica la direzione del flusso d'iniezione) e rappresentazione polare del MIL nelle direzioni di misura.

tando 513 orientazioni della griglia di misura ed 1000 linee per ciascuna rotazione.

Dal momento che per autovettori ed autovalori valgono rispettivamente le relazioni $\cos ^{2} \alpha+\cos ^{2} \beta+\cos ^{2} \gamma=1$ e $T_{1}+T_{2}+T_{3}=1$, viene introdotta una rappresentazione di questi parametri in grafici ternari, che hanno il vantaggio di consentire un'immediata visualizzazione delle caratteristiche della disposizione del rinforzo all'interno della matrice (Fig.11). Facendo riferimento sia alla Tab.2, sia alla Fig.11, appare evidente che i risultati presentano una dispersione minima, per cui nelle analisi successive si è ritenuto sufficiente ripetere 3 volte ciascuna misura. 

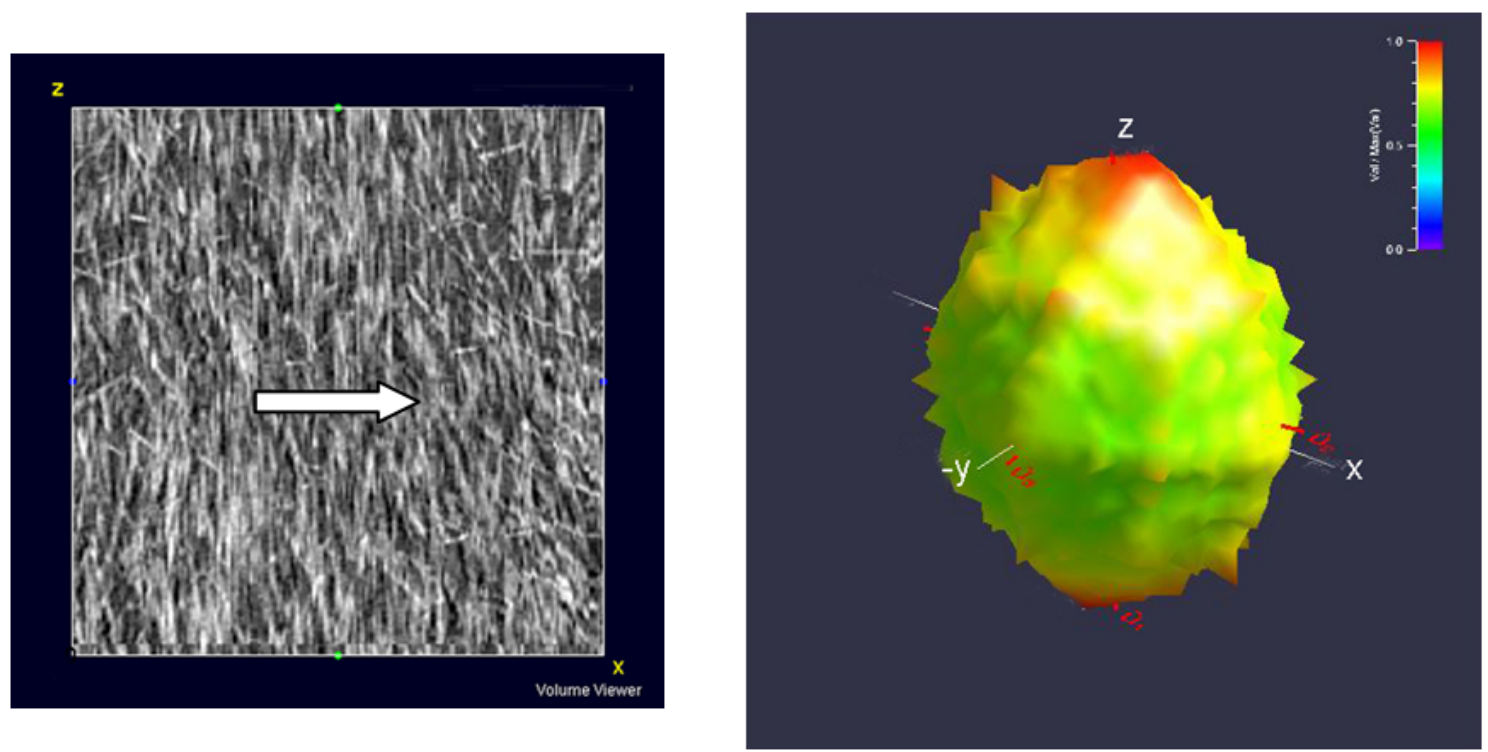

Figura 13: Zona core - Ricostruzione tomografica della distribuzione delle fibre (la freccia indica la direzione del flusso d'iniezione) e rappresentazione polare del MIL nelle direzioni di misura.

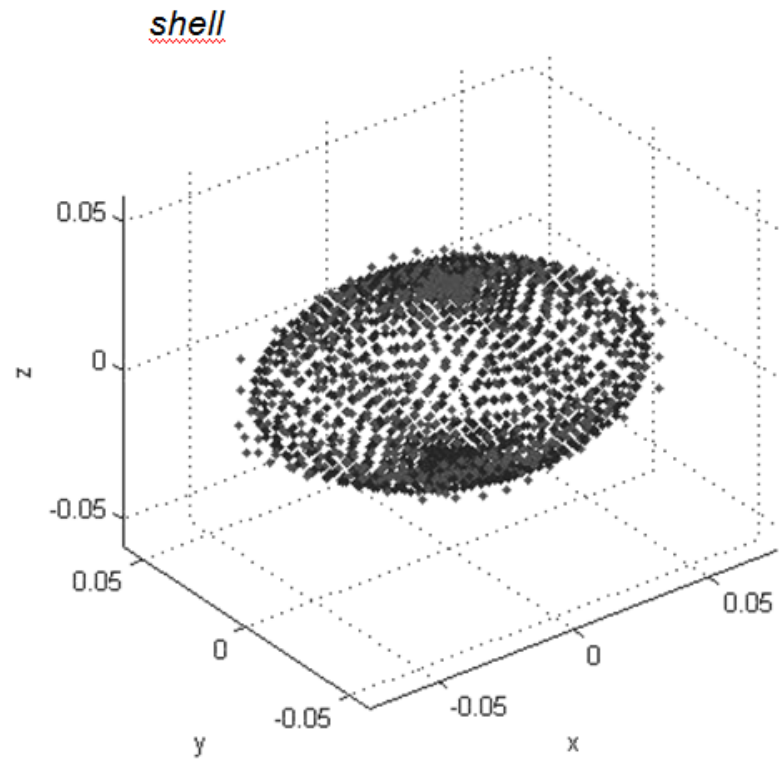

MIL

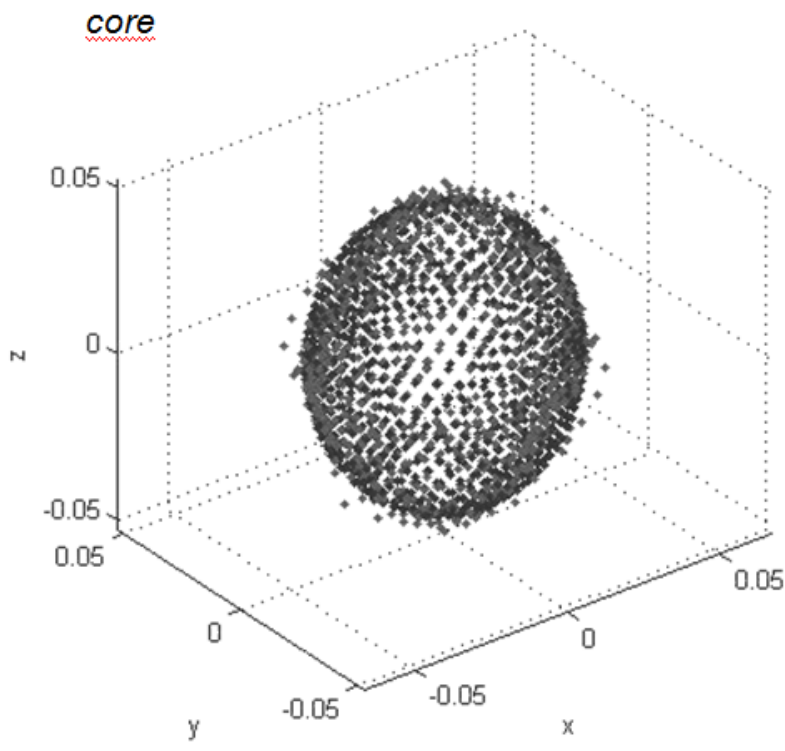

ellissoide

Figura 14: Rappresentazione grafica di MIL ed ellissoide del fabric tensor nelle direzioni di misura.

Come già citato, il software utilizzato consente di effettuare la misura per 513 o 2049 rotazioni. L'influenza del numero di orientazioni e di linee misura sul risultato ottenuto, valutata sullo stesso volume utilizzato in precedenza per valutare l'influenza della rotazione random, è riportata in Tab. 3 facendo sinteticamente riferimento al parametro Degree of Anisotropy, DA. Come nel caso precedente, la dispersione dei valori degli autovettori è talmente bassa da risultare non visualizzabile. Anche l'influenza dei parametri esaminati sul $D A$ risulta molto bassa, tranne nel caso di 513 orientazioni e 500 linee, cui corrisponde un campionamento insufficiente. Nella stessa tabella sono anche riportati i tempi di calcolo. Per le analisi successive sono state quindi adottate 513 orientazioni e 1000 linee di misura, che risulta essere la coppia di valori che ottimizza i tempi di calcolo a parità di risultato.

\section{RISULTATI E DISCUSSIONE}

I diagrammi polari di Figg.12 e 13 rappresentano i valori di MIL nelle 513 direzioni di misura in due volumi di 40 voxel di lato estratti rispettivamente nella zona shell e nella zona core del campione. La scelta di tale dimensione è dettata dallo spessore dello strato core. Le figure riportano anche le ricostruzioni delle fibre nelle zone analizzate. E' riconoscibile la distribuzione delle fibre già descritta nell'introduzione, corrispondente allo schema di 


\begin{tabular}{lcccccc} 
& DA & & & $\cos \boldsymbol{\alpha}$ & $\cos \boldsymbol{\beta}$ & $\cos \gamma$ \\
\hline shell & 1.82 & $T_{1}$ & 0.6160 & 0.9978 & 0.0650 & 0.0115 \\
\hline & & $T_{2}$ & 0.2599 & 0.0120 & -0.0082 & -0.9999 \\
\cline { 3 - 7 } & $T_{3}$ & 0.1241 & 0.0649 & -0.9979 & 0.0089 \\
\hline \multirow{2}{*}{ core } & 1.82 & $T_{1}$ & 0.4061 & 0.0898 & -0.0421 & 0.9951 \\
& & $T_{2}$ & 0.3495 & -0.0939 & -0.9950 & -0.0336 \\
\cline { 3 - 7 } & & $T_{3}$ & 0.2444 & 0.9915 & -0.0904 & -0.0933 \\
\hline
\end{tabular}

Tabella 4 - Autovalori e direzioni principali del fabric tensor.
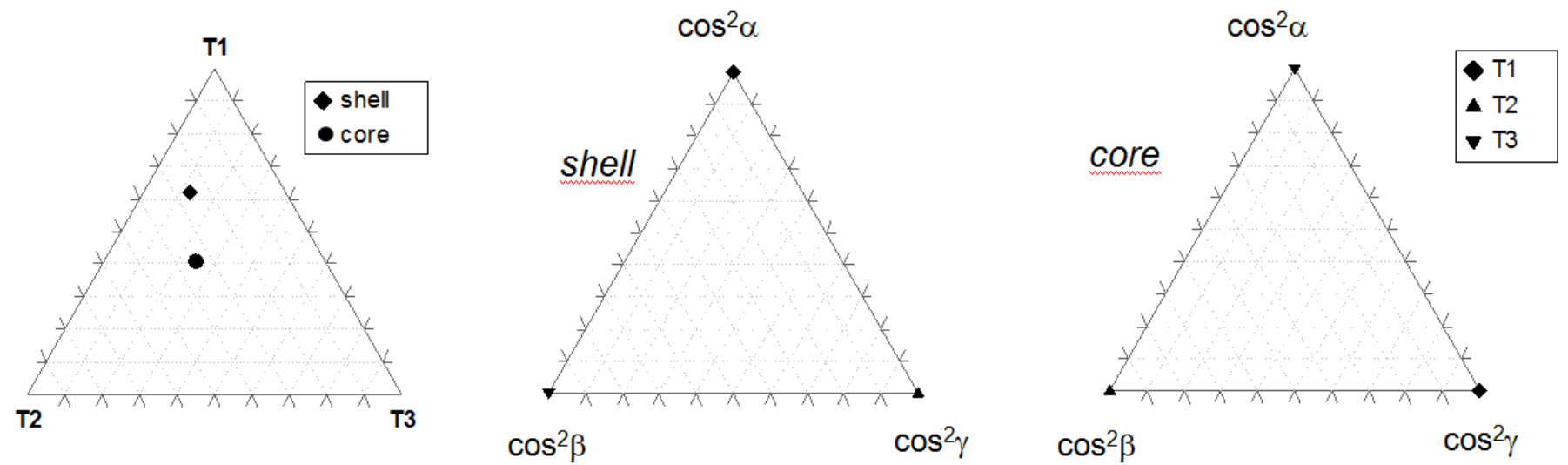

Figura 15: Rappresentazione su grafico ternario dei parametri del fabric tensor nelle zone shell e core.

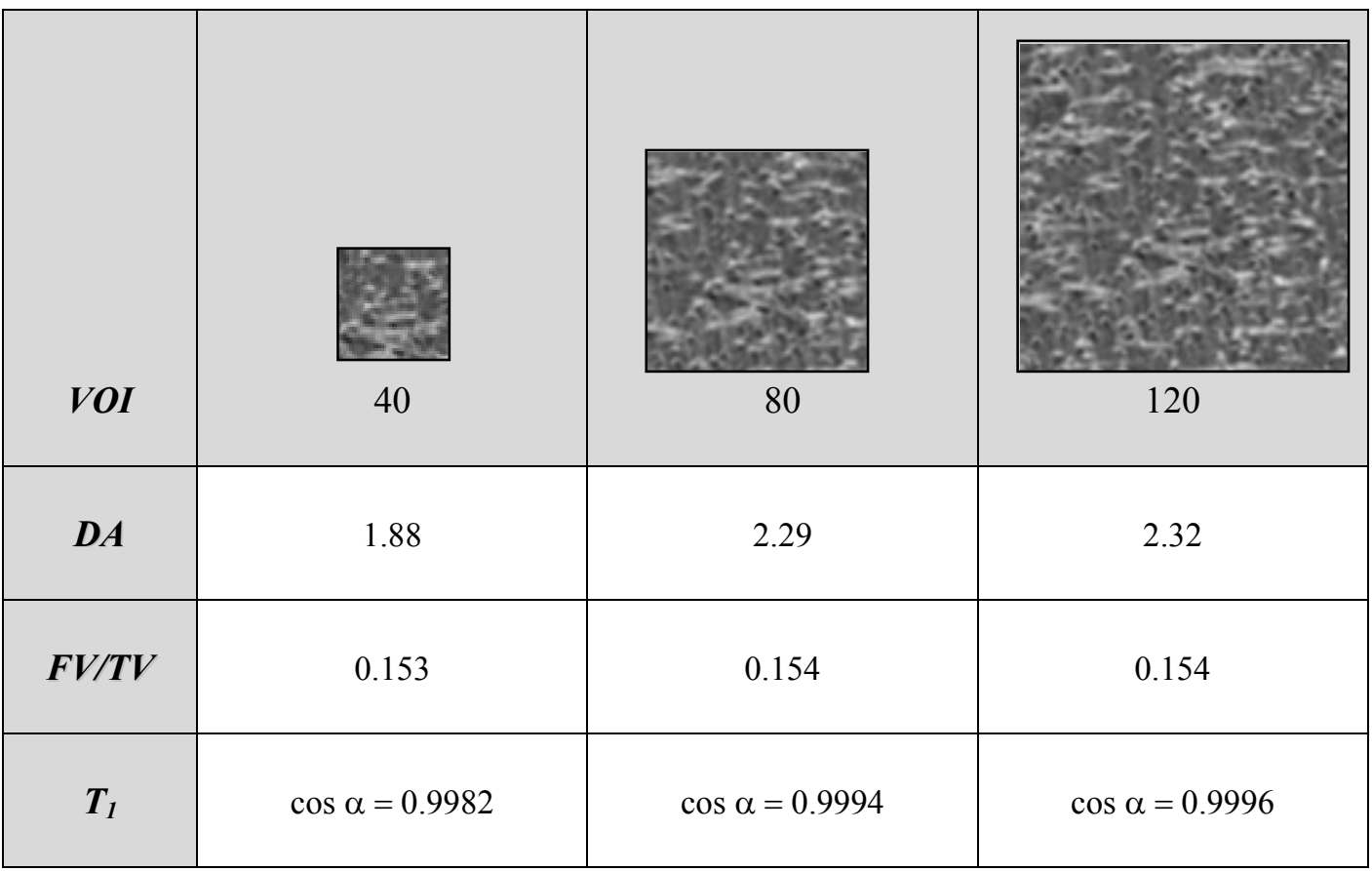

Tabella 5 - Influenza delle dimensioni del volume analizzato (VOI)

Fig. 5(a). Risulta pertanto confermato che la direzione preferenziale delle fibre nello strato di core è perpendicolare a quella delle fibre dello strato di shell, che a loro volta sono allineate al flusso d'iniezione.

Come già citato, per i materiali a due componenti è possibile approssimare il diagramma polare del MIL con il fabric tensor del MIL, rappresentabile mediante un ellissoide. La Fig.14 permette di apprezzare gli errori compiuti nel passaggio da $M I L$ all'ellissoide del fabric tensor.
I parametri del fabric tensor del MIL, ottenuti dalla media di 3 prove, sono riportati in Tab. 4 e, graficamente, in Fig.15. I valori degli autovettori (direzioni principali) del fabric tensor consentono di verificare che le fibre si dispongono nello strato shell secondo direzioni preferenziali ortogonali a quelle delle fibre nello strato core.

Dimensioni e posizione del volume analizzato

L'influenza delle dimensioni del volume analizzato (VOI) è stata valutata nella zona shell, di spessore mag- 


\begin{tabular}{l|c|c|c|c|} 
shell & VOI & $\mathbf{1 1}$ & $\mathbf{1 2}$ & $\mathbf{1 3}$ \\
\hline \multirow{5}{*}{ core } & $D A$ & 1.80 & 1.81 & 2.13 \\
\cline { 2 - 5 } & $F V / T V$ & 0.16 & 0.15 & 0.20 \\
\cline { 2 - 5 } & $T_{1}$ & $\cos \alpha=0.9990$ & $\cos \alpha=0.9982$ & $\cos \alpha=0.9979$ \\
\hline \multirow{5}{*}{$\boldsymbol{V O I}$} & $\mathbf{2 1}$ & $\mathbf{2 2}$ & $\mathbf{2 3}$ \\
\cline { 2 - 5 } & $D A$ & 1.45 & 1.21 & 1.4 \\
\cline { 2 - 5 } & $F V / T V$ & 0.23 & 0.20 & 0.18 \\
\cline { 2 - 5 } & $T_{1}$ & $\cos \gamma=0.9926$ & $\cos \gamma=0.9921$ & $\cos \gamma=0.9858$ \\
\hline
\end{tabular}

Tabella 6 - Influenza della posizione del volume analizzato (VOI).

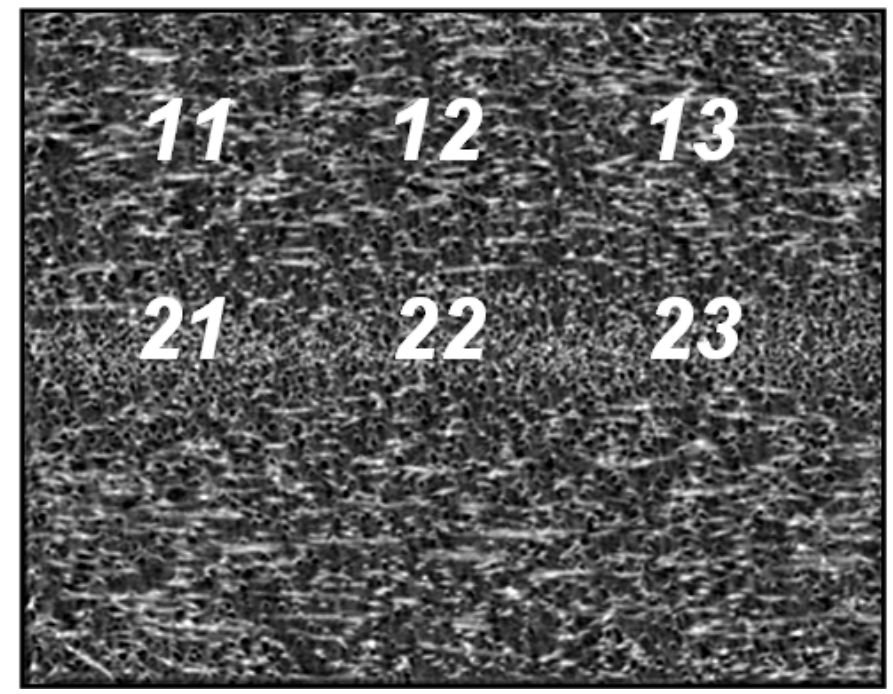

Figura 16: Posizione dei VOI nelle zone shell e core

giore rispetto alla zona core, utilizzando 3 volumi rispettivamente di lato 40, 80 e 120 voxel. I risultati sono riportati in Tab. 5 insieme ad una immagine dei volumi analizzati.

In Tab. 6 sono riportati i risultati ottenuti come media di 3 prove sui VOI di lato 40 voxel, estratti da zone shell e core come indicato in Fig.16. Questi risultati mostrano come sia possibile determinare il grado di anisotropia dei campioni e coglierne le variazioni locali, per mezzo di una caratterizzazione globale del volume esaminato, non fibra per fibra. In particolare, in Tab.5 è possibile osservare come al cambiare delle dimensioni del volume analizzato vi siano delle limitate variazioni del grado di anisotropia, per cui all'analisi di un volume di dimensione inferiore corrisponde una caratterizzazione particolarmente locale della struttura. Variazioni analoghe si riscontrano in Tab. 6, al variare della posizione di misura. E' comunque da notare come in ogni caso due zone shell e core, siano caratterizzate da valori del grado di anisotropia e direzioni preferenziali delle fibre nettamente diversi.

Gli sviluppi di questa ricerca avranno come obiettivo la valutazione delle relazioni che intercorrono tra distribuzione delle fibre e proprietà meccaniche di componenti realizzati in polimeri rinforzati con fibre corte. La determinazione della relazione tra costanti elastiche e autovalori e autovettori del fabric tensor non è immediata, in quanto le costanti elastiche sono descritte da un tensore del quarto ordine, mentre il fabric tensor è del secondo ordine. Cowin [20] ha sviluppato una formulazione per la determinazione delle costanti elastiche dell'osso trabecolare sano, la cui applicabilità al caso di polimeri rinforzati con fibre corte verrà verificata nel proseguimento della ricerca.

\section{CONCLUSIONI}

Le proprietà meccaniche dei materiali compositi rinforzati con fibre corte ottenuti per stampaggio a iniezione dipendono fortemente dalla distribuzione degli orientamenti delle fibre di rinforzo. Le tecniche comunemente impiegate per determinare l'orientamento delle fibre in questi materiali presentano limitazioni essenzialmente legate all'estrapolazione della distribuzione tridimensionale delle fibre a partire da immagini bidimensionali (radiografie o micrografie di sezioni). L'impiego della tomografia in contrasto di fase con luce di sincrotrone ha permesso la ricostruzione della distribuzione tridimensionale delle fibre in un campione di poliammide 6 rinforzato con il $30 \%$ di fibre corte di vetro. La successiva analisi dei volumi ricostruiti mediante un software che implementa la misura del MIL indica che è possibile determinare il grado di anisotropia dei campioni e coglierne le variazioni locali. Queste misure consentono una caratterizzazione globale del volume esaminato, non fibra per fibra. 
Gli sviluppi del lavoro presentato hanno come obiettivo la valutazione delle relazioni che intercorrono tra distribuzione delle fibre e le proprietà meccaniche di componenti realizzati in polimeri rinforzati con fibre corte.

\section{BIBLIOGRAFIA}

[1] S. Fu, B. Lauke, Effects of fiber length and fiber orientation distributions on the tensile strength of shortfiber-reinforced polymers. Composites Science and Technology, 56 (1996) 1179-1190.

[2] A. Bernasconi, P. Davoli, A. Basile, A. Filippi. Effect of fibre orientation on the fatigue behaviour of a short glass fibre reinforced polyamide-6. Int. J. Fatigue, 29 (2007) 199-208.

[3] C.M. Sonsino, E. Moosbrugger, Fatigue design of highly loaded short-glass-fibre reinforced polyamide parts in engine compartments. Int. J. of Fatigue (2007), doi: 10.1016/j.ijfatigue.2007.08.017.

[4] M Gupta, KK. Wang Fiber orientation and mechanical properties of short-fiber-reinforced injection-molded composites: simulated and experimental results. Polymer Composites ,14 (1993) 367-382.

[5] D. Hull, T.W. Clyne, An Introduction to Composite Materials $\left(2^{\text {nd }}\right.$ edition). Cambridge University Press (1996).

[6] S.G. Advani, C.L. Tucker, III, The use of tensors to describe and predict fiber orientation in short fiber composites. J. Rheology, 31 (1987) 751-784.

[7] R.S. Bay, C.L. Tucker III, Stereological measurement and error estimates for three-dimensional fiber orientation. Polymer Engineering and Science, 32 (1992) 240253.

[8] P.J. Hine, R.A. Duckett, Fiber orientation structures and mechanical properties of injection molded short glass fiber reinforced ribbed plates. Polymer Composites, 25 (2004) 237-254.
[9] A.R. Clarke, G. Archenhold, N.C. Davidson. A novel technique for determining the 3D spatial distribution of glass fibres in polymer composites. Composites Science and Technology, 55 (1995) 75-91.

[10] E.G. Kim, J.K. Park, S.H. Jo, A study on fiber orientation during the injection molding of fiber-reinforced polymeric composites (Comparison between image processing results and numerical simulation). J. of Materials Processing Technology, 111 (2001) 225-232.

[11] H. Shen, S. Nutt, D.Hull Direct observation and measurement of fiber architecture in short fiber-polymer composite foam through micro-CT imaging. Composites Science and Technology, 64 (2004) 2113-2120.

[12] R.A.Ketcham Three-dimensional grain fabric measurements using high-resolution X-ray computed tomography. J. of Structural Geology, 27 (2005) 1217-1228.

[13] S.C. Cowin, S.B. Doty, Tissue Mechanics, Sprinter, 2007.

[14] R.A.Lewis, Medical phase contrast x-ray imaging: current status and future prospects. Phys. Med. Biol., 49 (2004) 3573-3583.

[15] Arfelli et al., Mammography with synchrotron radiation: phase-detection techniques Radiology, 215 (2000) 286-293.

[16] A.C. Kak, M. Slaney: Principles of Computerized Tomographic Imaging, IEEE Press (1988).

[17] A. Odgaard, Three-Dimensional methods for quantification of cancellous bone architecture. Bone, 4 (1997) 315-328.

[18] G. M. Luo, A. M. Sadegh, S. C. Cowin, The mean intercept length polygons for systems of planar nets. J. of Materials Science, 26 (1991) 2389-2396.

[19] R. A. Ketcham, T. M.Ryan Quantification and visualization of anysotropy in trabecular bone. J. of Microscopy, 213 (2004) 158-171.

[20] S.C. Cowin, The relationship between the elasticity tensor and the fabric tensor. Mechanics of Materials, 4 (1985) 137-147. 